\title{
Frequency and water content dependencies of electrorheological properties
}

\author{
Weijia Wen, Hongru Ma, ${ }^{*}$ Wing Yim Tam, and Ping Sheng \\ Department of Physics, The Hong Kong University of Science and Technology, Clear Water Bay, Kowloon, Hong Kong
}

(Received 17 July 1996)

\begin{abstract}
The static yield stress and complex dielectric constant of glass spheres dispersed in vacuum oil are measured as a function of (applied electric field) frequency and water content. Based on a model in which the dielectric constants of the solid and the liquid constituents are given by the Debye form with a log-normal distribution of relaxation times, it is shown that the electrical and the mechanical electrorheological responses can be consistently explained through first-principles calculations. [S1063-651X(97)51102-0]
\end{abstract}

PACS number(s): 41.20.Cv, 61.90. $+\mathrm{d}, 62.20 .-\mathrm{x}$

Electrorheological (ER) fluids are a class of materials whose rheological properties are controllable by the application of an electric field. The discovery of this type of material was made by Winslow [1], who observed the ER effect with cornstarch suspended in oil. In the past decade, research in ER fluids has attracted much renewed interest. In particular, the role of water in producing the ER effect has been a widely debated topic [1-5]. For example, a recently proposed "water mechanism" is the so-called "conductivity effect" [2,6-13], where the presence of dc conductivity is key to the observed enhancement of ER yield stress in the presence of water. In particular, Felici et al. [8] and Conrad et al. $[9,11]$ have studied the conductivity effect in ER systems and analyzed the results in terms of a dielectric response function $\epsilon(\omega)=K(\omega)+i 4 \pi \sigma / \omega$, where $K(\omega)$ and $\sigma$ are real numbers. Here $\sigma$ is the dc conductivity of the system. In this work, we study the role of water in ER properties modifications in a system consisting of $1.5-\mu \mathrm{m}$ glass spheres dispersed in vacuum oil. By measuring the frequency dependencies of the static yield stress and the low-field complex dielectric constant as a function of increasing water content, and by calculating the same quantities through firstprinciples approaches, it is found that both the electrical and the mechanical electrorheological responses can be consistently explained on the basis of a model where the $K(\omega)$ part of the dielectric constants for both the solid and the liquid constituents of the ER fluids are frequency-dependent complex numbers given by the Debye form with a log-normal distribution of relaxation times. The dc conductivity term, $i 4 \pi \sigma / \omega$, can also be included in our model as an additional fitting parameter. However, best agreement between theory and experiments is found by setting the dc conductivity to zero. Our results therefore suggest that the presence of water essentially modifies the dielectric constants of the constituents from good insulators to that of poor insulators, given by the Debye form, and in the frequency range $\left(20-10^{5} \mathrm{~Hz}\right)$ we studied, the dc conductivity plays at most a very minor role as compared to the imaginary part of $K(\omega)$ in our analysis. The case where dc conductivity truly dominates, such as metallic particles suspended in oil, is also discussed and contrasted with the effect of water. It should be remarked that in

\footnotetext{
*Permanent address: Department of Physics, Jiaotung University, Shanghai, People's Republic of China
}

contrast to prior studies of the problems, our work distinguishes between the complex part of $K(\omega)$ and the dc conductivity effect, and employs first-principle calculations for our experimentally measured quantities. The resulting quantitative account of the experimental data based on the Debye model is noted to also rule out the "water bridge" mechanism [5] as a significant contributor (within the frequency range studied) to the yield stress in our system, where the water trapped in the contact areas between particles is held responsible for holding the particles together.

The ER fluids of our experiment consist of $1.5-\mu \mathrm{m}$ glass spheres suspended in vacuum oil. To prepare the samples, both the glass spheres and oil were heated at a temperature of $120{ }^{\circ} \mathrm{C}$ for about $24 \mathrm{~h}$ to remove any trace water. The dried particles were immediately placed in glass tubes, into which different amounts of water were injected with a pipette. The weights of the dried particle, and particles with water added, were measured by an electronic balance (OHAUS CORP. AP250D), from which the water contents $w$ were calculated. The water contents of the three samples 1,2, and 3 are $0.04979,0.07986$, and 0.11441 , respectively, in weight fraction of glass spheres. The mouths of the tube were sealed and then stored at a temperature of $120^{\circ} \mathrm{C}$ for $10 \mathrm{~h}$. The tubes were rotated continuously to ensure uniform vaporization. After cooling to room temperature, a measured amount of oil was injected into the tube, and the hydronic particles were uniformly immersed in oil through the application of ultrasonic vibrations, with a volume fraction of about $9 \%$.

With a cell comprised of two parallel plate electrodes, the dielectric measurements of the ER fluids were performed by an HP4284A LCR meter in a frequency range of $20 \mathrm{~Hz}$ to $100 \mathrm{kHz}$. The static yield stress was measured by using a standard parallel plate torsional device with a root-meansquare (RMS) electric field of $510 \mathrm{~V} / \mathrm{mm}$ applied across the ER fluids sandwiched between the two parallel plates. The lower plate was rotated slowly, dragging the top plate with a torque, which was connected via a torque meter to the top plate. The static yield stress was read out when slipping occurred between the two plates, after subtracting off the zerofield value (which is about $1 \%$ of the high-field value). The static yield stress was observed to have an accurate $E^{2}$ field strength dependence.

In Figs. 1 and 2, the measured real and imaginary parts of the dielectric constants are plotted as a function of frequency for the three samples with increasing amount of water. In 


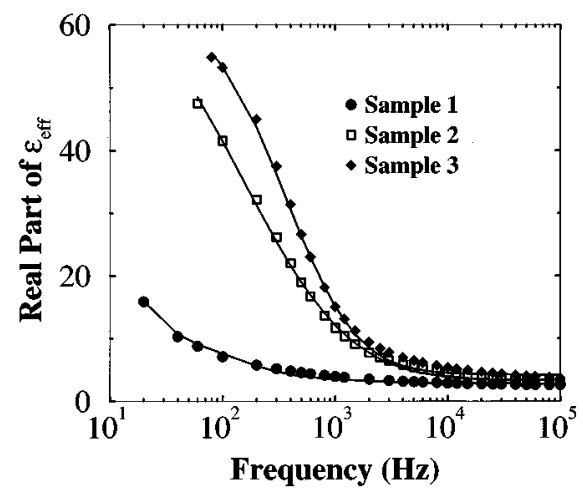

FIG. 1. The real part of effective dielectric constant of the three samples plotted as a function of frequency. The symbols represent the experimental measurements and lines are fitted theoretical calculations.

Fig. 3, the measured static yield stresses for the same three samples are plotted as a function of frequency. It is seen that the water content has an important effect on the frequency dependencies of the complex dielectric constant and the static yield stress. To explain these experimental results, we use the following formalism for the calculation of the static yield stress and the dielectric constant.

We model the ER fluid as a two-component system consisting of spherical solid particles of radius $R$ and complex dielectric constant $\varepsilon_{1}$ dispersed in a liquid characterized by $\varepsilon_{2}$. The electrostatic problem to be solved is given by

$$
\boldsymbol{\nabla} \cdot\left[1-\frac{1}{s} \eta(\mathbf{r})\right] \boldsymbol{\nabla} \phi=0
$$

where $s=\varepsilon_{2} /\left(\varepsilon_{2}-\varepsilon_{1}\right)$ is the only relevant material parameter in the problem. Here $\phi$ is the electrical potential and $\eta(\mathbf{r})$ is the characteristic function of the solid component, defined as having the value 1 at those spatial points occupied by the solid particles, and zero otherwise. The formal solution of Eq. (1), given the condition of $\Delta \phi / l=E=1$ in the $z$ direction, can be expressed in the operator notation as

$$
\phi=-s \frac{z}{s-\Gamma},
$$

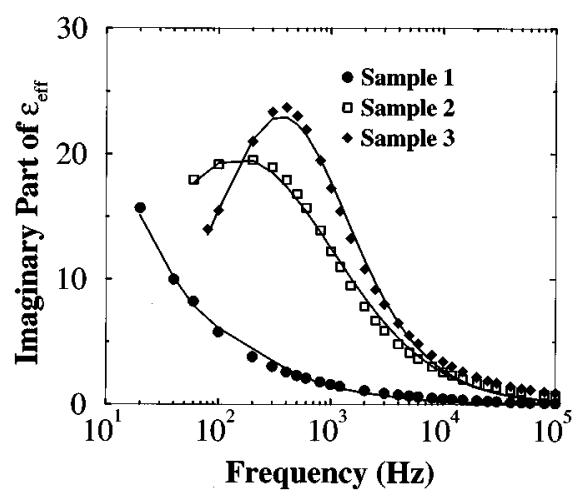

FIG. 2. The imaginary part of effective dielectric constant of the three samples plotted as a function of frequency. The symbols represent the experimental measurements and lines are fitted theoretical calculations.

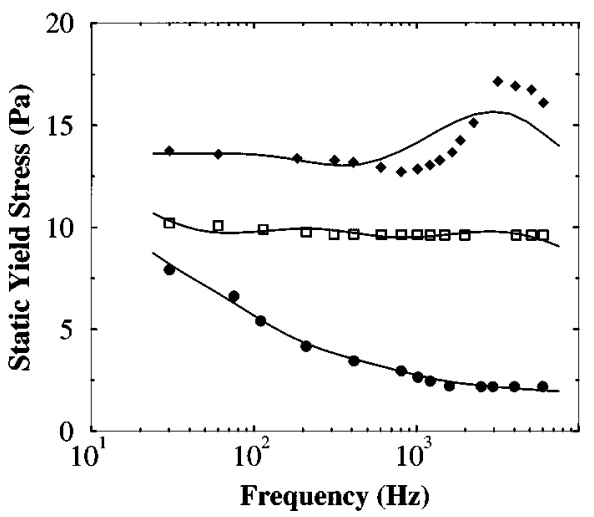

FIG. 3. The measured (symbols) and fitted (lines) static yield stress plotted as a function of frequency. From bottom to top: sample 1, sample 2, and sample 3.

where

$$
\Gamma=\frac{1}{V} \int d \mathbf{r}^{\prime} \eta\left(\mathbf{r}^{\prime}\right) \nabla^{\prime} G_{0}\left(\mathbf{r}-\mathbf{r}^{\prime}\right) \cdot \nabla^{\prime}
$$

is an integral-differential operator, with $G_{0}\left(\mathbf{r}-\mathbf{r}^{\prime}\right)$ $=1 / 4 \pi\left|\mathbf{r}-\mathbf{r}^{\prime}\right|$ denoting the Green's function for the Laplace equation, and $V$ the sample volume. By defining the inner product operation as

$$
\langle\phi \mid \psi\rangle=\int d \mathbf{r} \eta(\mathbf{r}) \boldsymbol{\nabla} \phi(\mathbf{r})^{*} \cdot \nabla \psi(\mathbf{r}),
$$

it becomes possible to write the effective dielectric constant as

$$
\frac{\bar{\epsilon}_{z z}}{\epsilon_{2}}=-\frac{1}{V} \int d \mathbf{r}\left(1-\frac{1}{s} \eta(\mathbf{r})\right) \frac{\partial \phi}{\partial z}=1+\frac{1}{s}\langle z \mid \phi\rangle \frac{1}{V} .
$$

From Eqs. (2) and (5), it follows that the effective dielectric constant is given by the Bergman-Milton representation [14]:

$$
\frac{\bar{\epsilon}_{z z}}{\epsilon_{2}}=1-\frac{1}{V} \sum_{n} \frac{f_{n}}{s-s_{n}} .
$$

Here $f_{n}=\left|\left\langle z \mid \phi_{n}\right\rangle\right|^{2}$, and $s_{n}$ and $\phi_{n}$ are the $n$th eigenvalue and eigenfunction of the operator $\Gamma$.

Since ER systems are always operated at frequencies $<10^{5} \mathrm{~Hz}$, the long-wavelength limit is valid. Therefore, the free energy density of the system is given by [15]

$$
f=-\frac{1}{8 \pi} \operatorname{Re}\left(\bar{\epsilon}_{z z}\right) E_{0}^{2}-S T,
$$

where $E_{0}$ is the average (or applied) electric field, $S$ is the entropy, and $T$ is temperature. It should be noted that in Eq. (7), where $\bar{\epsilon}_{z z}$ is complex, its imaginary part relates to the dissipation of the system. However, as long as heating of the system is not excessive, the condition of lowest free energy should be operative. This is generally the case in most physical systems. It follows that in the limit of small applied field, the ground state of the ER fluids is clearly the one that has the highest entropy, i.e., the randomly dispersed configuration. This is the state in which the complex dielectric con- 
stant of the system is measured. In the high-field limit, the entropy part of the free energy may be neglected, and the particle structure that gives the largest effective dielectric constant, or the lowest free energy density, was first proposed by Tao and Sun [16] to be a body-centered-tetragonal (BCT) structure based on dipole interactions. This ground state structure was later confirmed by various authors [1720] with more accurate calculations. The static yield stress was measured at the high-field state. Unfortunately, we have yet been unable to systematically measure the dielectric constants of the high-field state.

To calculate the static yield stress of an ER fluid, it is necessary to perturb away from the equilibrium state by applying a shear deformation to the system that is perpendicular to the applied field. The shear deformation basically has two components, i.e., each chain is tilted with a shear angle $\theta$ with respect to the field direction, and thereby also elongated; i.e., the particles are separated as a result. To determine the static yield stress, the free energy density is first calculated as a function of shearing angle. The stress is then obtained by numerical differentiation of the free energy density as a function of the shearing angle $\theta$. The static yield stress is defined to be the maximum stress beyond which the stress decreases with increasing strain, i.e., the solid structure becomes unstable. This is described in more detail elsewhere [20].

We model the dielectric constants of the solid particles and the liquid by a Debye form with a log-normal distribution of relaxation times,

$$
\varepsilon-\varepsilon_{\infty}=\int d \tau P(\tau) \frac{\varepsilon_{\Delta}}{1-i \omega \tau}
$$

where

$$
P(\tau)=\frac{1}{\sqrt{2 \pi} \Delta \tau} \exp \left[-\frac{\left(\ln \tau / \tau_{0}\right)^{2}}{2 \Delta^{2}}\right]
$$

$\varepsilon_{\infty}$ is the high frequency dielectric constant, $\varepsilon_{\Delta}$ is the difference between static and high frequency dielectric constant, $\omega$ is the frequency of the applied field, and $\tau$ is the Debye relaxation time. $\Delta$ and $\tau_{0}$ are two parameters of the lognormal distribution.

Since the ER system before the application of a high electric field has the random dispersion microstructure, its effective complex dielectric constant at low volume concentration may be calculated by the Maxwell-Garnett formula [21] with a high degree of accuracy:

$$
\frac{\varepsilon_{\mathrm{eff}}-\varepsilon_{2}}{\varepsilon_{\mathrm{eff}}+2 \varepsilon_{2}}=p \frac{\varepsilon_{1}-\varepsilon_{2}}{\varepsilon_{1}+2 \varepsilon_{2}} .
$$

Here the subscripts 1, 2, and eff stand for particle, liquid, and effective, respectively; $p$ is the volume fraction of spheres. Because the volume fraction is small (9\%) in our experiment, the effective dielectric constant is more sensitive to the dielectric constant of the liquid as can be calculated from Eq. (10). In contrast, the static yield stress, which is measured under high applied field and the particles are in close proximity to each other, is sensitive to the dielectric constant of the spheres. Based on this observation, we have
TABLE I. The fitted parameters for the three samples. $P$ and $L$ stands for particles and liquid, respectively.

\begin{tabular}{lccccc}
\hline \hline & & $\varepsilon_{\infty}$ & $\varepsilon_{\Delta}$ & $\tau_{0}(\mathrm{~s})$ & $\Delta$ \\
\hline \multirow{3}{*}{ Sample 1 } & $P$ & 62.7 & 789 & $2.92 \times 10^{-2}$ & 2.89 \\
& $L$ & 2.2 & 273 & 4.52 & 3.44 \\
Sample 2 & $P$ & 247 & 561 & $3.52 \times 10^{-2}$ & 4.39 \\
& $L$ & 2.6 & 49.8 & $1.07 \times 10^{-3}$ & 1.49 \\
Sample 3 & $P$ & 422. & 123 & $6.25 \times 10^{-4}$ & $\sim 0$ \\
& $L$ & 3.3 & 44.6 & $4.44 \times 10^{-4}$ & .832 \\
\hline \hline
\end{tabular}

fitted the parameters of the spheres and the liquid in an iterative manner in order to converge to the optimal fit. That is, we first determined the $\varepsilon_{\infty}$ of the liquid from the measured high frequency effective dielectric constant. Then with a reasonable guess for the dielectric constant of the spheres, we fit the remaining three parameters of the liquid dielectric constant to the measured frequency dependence of the effective dielectric constant. Then by treating the liquid dielectric constant as known, we fit the four parameters for the dielectric constant of the solid spheres to the measured frequency variation of the yield stress. The three parameters of the liquid dielectric constant are then refitted, and this iteration is continued until convergence results. The resulting parameter values are given in Table I. It should be noted that while there are a total of seven fitting parameters for the three curves of each sample, four of those parameters are related to the distribution functions of the relaxation times, and therefore can only affect the results in a very constrained manner [22]. That is, the good agreement between theory and experiments as seen from the two lower-water-content samples, and the consistency that is achieved between two different types of properties, reflect the validity of the Debye model and yield the distributions of relaxation times as a result. The fit for the yield stress of the third sample is less impressive than the other two. Nevertheless, the theory does give correct qualitative behavior. It should be noted that in the Debye model the dc conductivity is zero. We have tried to insert an additional term in the dielectric constants of solid particles and the liquid, $i 4 \pi \sigma / \omega$, to reflect the presence of the dc conductivity. However, our fitting process yields zero as the final value for that term, i.e., there is no dc conductivity effect. In fact, a separate experiment had been carried out by using metallic spheres and insulated electrodes, where the dc conductivity truly dominates. It was seen that the spheres do not form columns. Instead, they form a fractal-like pattern. A little thought on this result led to the recognition that the presence of particle dc conductivity essentially modified the electrodes from planar to irregular shapes, with sharp corners (single particle) where the electric field is the highest. The situation then becomes similar to that of diffusion-limited aggregation, and the formation of a fractal-like pattern is therefore not surprising. Details of the metallic-particle experiment will be reported elsewhere [23]. The point here, though, is that if the particle dc conductivity were indeed dominant, its effect should be easily visible as in the metallic case.

A plausible physical picture that emerges from our results is that the water phase exists as droplets in oil, and the distribution in relaxation times reflects the distribution in the 
size of the droplets, though the precise connection is still to be determined. At low water contents, the droplets tend to aggregate at the surfaces of the spheres, leaving the oil relatively free of water. That is evidenced by the fact that the dielectric constant of the liquid phase (consisting of oil plus water droplets) is very close to that of oil at low water content, though that of the solid phase is very different. At higher water content, however, the droplets could be dispersed in the oil as well, thus affecting the dielectric constant of the liquid phase.
In conclusion, we have measured the frequencydependent effective dielectric constant and yield stress of glass or vacuum oil with different water contents. The measured results are found to be quantitatively explainable on the basis of a model in which both the dielectric constants of solid and liquid are given by the Debye form, with a lognormal distribution of the relaxation times.

We wish to acknowledge the support of HKUST Research Infrastructure Grant RI93/94.SC09 for this work.
[1] W. M. Winslow,J. Appl. Phys. 20, 1137 (1949).

[2] F. E. Filisko and L. H. Radzilowski,J. Rheol. 34, 539 (1990).

[3] T. N. Tombs and T. B. Jones,IEEE Trans. Ind. Appl. 29, 281 (1993).

[4] Y. Z. Xu and R. F. Liang,J. Rheol. 35, 1355 (1991).

[5] H. See, H. Tamura and M. Doi,J. Phys. D 26, 746 (1993).

[6] R. A. Anderson, in Electrorheological Fluids, edited by R. Tao (World Scientific, Singapore, 1992), p. 81.

[7] L. C. Davis,Appl. Phys. Lett. 60, 319 (1992); J. Appl. Phys. 72, 1334 (1992); L. C. Davis, ibid. 73, 680 (1993).

[8] N. Felici, J. N. Foulc, and P. Atten, in Electrorheological Flu$i d s$, edited by R. Tao and G. D. Roy (World Scientific, Singapore, 1994), p.139.

[9] H. Conrad and Y. Chen, in Progress in Electrorheology, edited by K. O. Havelka and F. E. Filisko (Plenum Press, New York, 1995), p. 55.

[10] D. A. Brooks, in Progress in Electrorheology (Ref. [9]), p. 87.

[11] X. Tang, C. Wu, and H. Conrad,J. Rheol. 39, 1059 (1995); J. Appl. Phys. 78, 4183 (1995).

[12] K. Lu, W. Wen, C. Li, and S. Xie,Phys. Rev. E 52, 6329 (1995).

[13] W. Wen and K. Lu,Appl. Phys. Lett. 68, 3659 (1996).

[14] D. J. Bergman, in Solid State Physics Vol. 46, edited by H. Ehrenreich and D. Turnbull (Academic Press, New York, 1992), p. 147; G. W. Milton,Appl. Phys. A 26, 1207 (1981); G.
W. Milton,J. Appl. Phys. 52, 5286 (1980).

[15] L. D. Landau and E. M. Lifshiz, Electrodynamics of Continuous Media, 2nd ed. (Pergmon Press, New York, 1984), p. 50.

[16] R. Tao and J. M. Sun,Phys. Rev. Lett. 67, 398 (1991); Phys. Rev. A 44, R6181 (1991).

[17] G. Bossis, H. Clercx, Y. Grasselli, and E. Lemaice, in Electrorheological Fluids (Ref. [8]), p. 153; H. Clercx and G. Bossis,Phys. Rev. E 48, 2721 (1993).

[18] Richard Friedberg and Y. K. Yu,Phys. Rev. B 46, 6582 (1992).

[19] L. C. Davis,Phys. Rev. A 46, R719 (1992).

[20] H. Ma, W. Wen, W. Y. Tam, and P. Sheng, Phys. Rev. Lett. (to be published). Here we wish to note that in terms of the calculated yield stress, the use of the BCT ground state or the (actually observed) columnar structure results in very small differences. This is because the difference between the two states lies in the surface energy, which is only $10^{-3}$ times of the total energy. This has been discussed in our work quoted above.

[21] Ping Sheng, Introduction to Wave Scattering, Localization, and Mesoscopic Phenomena (Academic Press, New York, 1995), Chap. 3.

[22] The fit to the experimental results is sensitive to the form of the distribution. We have tried six forms other than the lognormal distribution. All give poor fit.

[23] W. Wen and K. Lu, Phys. Fluids (to be published). 